Rabaska

Revue d'ethnologie de l'Amérique française

\title{
Table ronde sur le conte
}

\section{Christian-Marie Pons}

Volume 2, 2004

URI : https://id.erudit.org/iderudit/201656ar

DOI : https://doi.org/10.7202/201656ar

Aller au sommaire du numéro

Éditeur(s)

Société québécoise d'ethnologie

ISSN

1703-7433 (imprimé)

1916-7350 (numérique)

Découvrir la revue

Citer ce compte rendu

Pons, C.-M. (2004). Compte rendu de [Table ronde sur le conte]. Rabaska, 2,

185-193. https://doi.org/10.7202/201656ar d'utilisation que vous pouvez consulter en ligne.

https://apropos.erudit.org/fr/usagers/politique-dutilisation/ 


\title{
Table ronde sur le conte
}

\author{
Christian-Marie Pons
}

Conseil québécois du patrimoine vivant, Québec

Le Conseil québécois du patrimoine vivant (CQPV) a tenu à Québec, le 14 novembre 2003, une table de concertation sectorielle portant sur le conte. Elle réunissait une douzaine de conteurs et organisateurs de Montréal et de Hull, de l'Estrie, de la région de Québec, de Charlevoix et du Bas-du-fleuve. Elle a été l'occasion, pendant deux heures et quart, d'échanges dynamiques sur des sujets parfois délicats et importants sur le devenir du conte, toujours avec complicité et bonne humeur.

Christian-Marie Pons, chargé d'animer la table, ouvre celle-ci en remerciant le $\mathrm{CQPV}$ de son accueil et en introduisant un bref rappel historique pour situer le présent exercice : une première table de concertation (CQPV, Québec, 25 janvier 2001) au cours de laquelle fut, notamment, soulevée la question d'une association des conteurs et qui fut conclue par une suspension de la question ; une seconde table de concertation (CQPV, Montréal, novembre 2002) au cours de laquelle fut annoncée la reprise du projet de regroupement du conte et qui fut conclue par la demande auprès du CQPV de suspendre toute décision et toute action concernant le conte, le temps que le milieu du conte s'organise et prenne position.

C.-M. Pons poursuit ce rappel historique en mentionnant qu'une première assemblée de conteurs et d'organisateurs, réunissant une cinquantaine de personnes, s'était tenue le 5 avril 2003 à Montréal et à l'issue de laquelle deux décisions furent prises : d'une part, le souhait unanime qu'un regroupement du conte au Québec soit finalement constitué ; d'autre part, l'élection d'un collège de cinq personnes (Mike Burns, Jacques Falquet, JeanMarc Massie, Éveline Ménard et Christian-Marie Pons) mandatées comme administrateurs provisoires pour assurer les démarches nécessaires à la mise sur pied du dit regroupement. Une assemblée de fondation, réunissant une quarantaine de personnes du milieu du conte, s'est tenue le 20 octobre 2003, à Montréal, au cours de laquelle furent adoptés à l'unanimité la constitution, les objets et les règlements généraux du Regroupement du conte au Québec (RcQ) et fut nommé un premier conseil d'administration composé de sept personnes (Jean-Marc Massie, président ; Petronella van Dijk, viceprésidente ; Jacques Falquet, secrétaire ; Mike Burns, trésorier ; Danielle Brabant, Renée Robitaille et Maurice Vaney, administrateurs). 
Pons conclut son introduction en soulignant la fulgurante évolution de la pratique du conte, de son état et de son milieu. Il propose de consacrer la présente table à l'évaluation, dans un premier temps sous forme de bilans et de perspectives, de cette impressionnante mutation du conte au Québec afin de permettre, dans un second temps, de dégager la nature spécifique du conte au sein du patrimoine vivant et, ultimement, de préciser la place et les relations entre le nouveau Regroupement du conte au Québec vis-à-vis du Conseil québécois du patrimoine vivant.

\section{Mise en contexte}

Pons soumet, en préambule et pour démarrer la discussion, un ensemble de réflexions, synthèse et constat des « enjeux actuels du conte au Québec ». Il articule son propos autour d'un double niveau, quantitatif et qualitatif, et précise les marques de vitalité et de fragilité, les forces et les faiblesses du phénomène du conte aujourd'hui.

Quantitativement, les forces : Pons souligne la croissance phénoménale des facteurs et activités du conte, tant en termes de conteurs et de relève, qu'en termes de lieux et d'événements répandus un peu partout au Québec, qu'en termes de publics, plus nombreux, plus diversifiés. Il insiste sur la reconnaissance même du phénomène, très marginal il y a à peine cinq ans, beaucoup plus présent et visible dans l'actuel paysage culturel, tant auprès des organismes subventionnaires, identifiant de plus en plus le conte comme pratique autonome et distincte, qu'auprès des écoles et entreprises, des médias; sans omettre une reconnaissance internationale (circulation des conteurs du Québec à l'étranger et attrait des conteurs étrangers à se produire au Québec). Force encore par l'organisation du milieu, témoin la mise sur pied du récent Regroupement du conte au Québec. Premier bilan positif, réel et solide, à conserver.

Quantitativement, les faiblesses, les fragilités, voire les inquiétudes : les manques de financement à venir tant du côté provincial que bientôt du fédéral sont à craindre, les coupures dans le budget culturel ont déjà commencé. Le constat à vérifier d'un certain essoufflement (saturation ?) des publics ; plusieurs organisateurs ont constaté une certaine stabilisation, voire diminution de la fréquentation des spectacles et événements. La multiplicité, notamment cet automne, d'événements dédiés au conte risque un « embouteillage » et une dilution des énergies : le succès est parfois cause difficile à prévenir de déboires à venir. La prolifération de nouveaux conteurs sur le marché peut aussi devenir source d'engorgement.

Qualitativement, les points forts : la spécificité du conte comme pratique socioculturelle est de mieux en mieux assumée et reconnue ; on cesse de confondre le conte avec un jeu d'enfant ou comme seule nostalgie d'un bon 
vieux temps ; on situe mieux le conte comme réalité vivante, contemporaine mais gardienne de valeurs qui autrement ont tendance à disparaître (le partage, la proximité, la chaleur humaine, le plaisir du récit direct, de la rencontre, etc.). La légèreté du dispositif du contage, sa souplesse, favorise la survie du conte aux dépens d'industries plus massives, plus onéreuses. La stimulation entre conteurs et face à des publics plus avertis incite à développer de nouveaux récits, de nouvelles façons de conter, d'aller plus loin, d'innover. L'originalité même, comme piste à poursuivre, dans les deux sens du mot originalité : original, par l'invention, la création, l'appropriation individuelle de récits collectifs ; original, par la retrouvaille de ce qui fait la spécificité et la qualité du conte comme moyen de partage et d'échange, et qui a fait ses preuves avec le temps.

Qualitativement, les fragilités, les risques que la qualité s'affadisse dans la quantité : le succès du « conte-spectacle », parfois plus soucieux d'occuper le public que de s'occuper de " poésie ». Les risques du showbiz, la tentation d'alourdir le conte par sa théâtralisation et la perte d'une certaine spontanéité. La légèreté du dispositif est un atout, celle des contenus peut devenir misère : le conte va-t-il mourir de rire ? Va-t-il se contenter de divertir ? Le conte n'est pas à l'abri des effets de « macdonalisation » et de fast-food culturel. À l'essoufflement des publics, prévoir celui des conteurs : usure d'un même répertoire trop souvent exposé ; un conte peut se redire et se répéter, mais il y a des limites; le conte s'adapte mal au rythme de la consommation. L'absence de critique, enfin, d'un milieu exigu, le risque de complaisance ; peut-être est-il temps d'une certaine exigence : raconter n'est pas toujours conter; dans l'effervescence, attention à la précipitation à vouloir chercher la reconnaissance avant la connaissance et le savoir-faire du conteur ; l'apparente facilité d'un terrain maintenant défriché peut tenter de vouloir arriver trop vite; la formation est quasi inexistante au Québec ; on peut regretter, face à certaines prestations, le manque de maturité, de maturation : le conte est souvent servi comme un vin trop jeune. Les modes passent; le conte serait-il une mode, finalement?

Ce propos introductif veut favoriser le bilan et le débat, non démobiliser ; son souci, bien sûr est de provoquer la réflexion et le constat pour mieux prévenir et améliorer cet état du conte.

\section{Discussions}

Les participants sont invités à réagir, à commenter, à discuter à partir de ce portrait initial. Plusieurs points vont être soulevés par les personnes présentes. Une première réaction est celle de modérer le bilan de départ; le constat établi est bien réel, mais il fait plus «l'objet de préoccupations que d'inquiétudes » (Yves RobitallLe). 


\section{Les publics}

La saturation du public est davantage la perception d'un observateur assidu et qui suit de près et régulièrement les manifestations du conte que celle d'un public plus occasionnel. Hypothèse d'un rythme différent entre une relative concentration du conte à Montréal et celle plus diluée qu'on retrouve en région, où le public serait encore à conquérir : l'époque du " défrichage » est toujours d'actualité. ANDRÉ LEMELIN soulève une différence entre « public » (celui qui connaît déjà l'existence du conte et se déplace pour l'écouter ) et « marché » (vaste potentiel non rejoint par le conte et auprès duquel il y a encore lieu de promouvoir le conte, les écoles et les entreprises par exemple). Lemelin distingue aussi la notion de «plafond », clos une fois atteint, et celle de « plateau » au-delà, à poursuivre après l'avoir atteint; après cinq ans de développement, le conte a acquis un certain rythme. La diminution ressentie des publics tient aussi sans doute au fait d'un manque de promotion, faute de moyens.

JEAN-MARC MASSIE insiste sur la responsabilité des organisateurs pour dynamiser et hausser le milieu du conte et son niveau de qualité, d'un côté en spécifiant les événements proposés (diversifier, donner une couleur particulière compte tenu des exigences particulières d'un public), d'un autre côté en assumant la part ingrate d'une sélection des conteurs en fonction des intentions, de la " vision » à donner aux événements prévus. Après une première période d'émergence, l'industrie du conte aurait atteint une période de consolidation, où critique et sélection sont devenues nécessaires.

\section{Les lieux}

La notion de lieu (de spectacle, de diffusion) apparaît cruciale, et pour différentes raisons : DANIELLE BRABANT suggère le fait de favoriser de nouveaux lieux d'expression du conte afin d'y rejoindre de nouveaux publics, d'autres milieux à découvrir. Nouveaux lieux, à la fois à l'écart des lieux déjà institués et réputés comme lieux de conte (par exemple, le Sergent recruteur et l'Intrus, à Montréal ; le Fou Bar à Québec), à la fois comme lieux physiquement et fonctionnellement différents (par exemple, développement des ballades contées, en extérieur, évocation d'expériences tentées : conte en cuisine, à Saint-Élie, ou à bord d'autobus, en France). ANDRÉ LEMELIN signale de nouvelles dynamiques à ne pas négliger : celle, convenue, d'un lieu institué vers lequel le public se rend entendre un conteur, mais celle aussi où le conteur se déplace là où se trouve un public potentiel (écoles, entreprises, voire des lieux privés ou semi-privés). BERNARD GrondIN appuie l'idée en rappelant que la meilleure promotion du conte est celle du conteur qui se rend là où sont les gens, dans leur quotidien et proche d'eux, retrouvant ainsi un des procédés traditionnels du conteur qui se rendait dans les maisons plutôt que 
d'attendre un public dans une salle de spectacle. Lemelin ajoute cependant qu'il est difficile d'agir ainsi en ville dont l'individualisme est dominant, et qu'un travail de promotion reste essentiel.

JEAN-MARC MASSIE confirme cette importance du lieu et son impact sur la présence du public : un lieu bien identifié, réputé comme espace de conte et attrayant stimule l'intérêt du public et sa venue (c'est le cas, par exemple du Sergent recruteur); en revanche un événement, un festival, qui se produit dans trop de lieux différents et non identifiés au conte affaiblit ses chances d'attirer une clientèle et plus encore de la fidéliser. DENIS TRUDEL souligne le fait qu'il y a des bons et des mauvais lieux pour le conte, question d'atmosphère, même s'il est très difficile de définir d'emblée ce qui fait qu'un lieu soit bon ou mauvais, attirant ou non. Un intervenant rappelle aussi la nécessité que le conteur invité dans un lieu sache clairement à quoi s'attendre, et qu'il est de la responsabilité de l'organisateur autant que du conteur de bien préciser les conditions de contage (par exemple, la nécessité ou non d'avoir à utiliser un micro).

Après avoir relevé un ensemble d'éléments de type factuel, mais déterminants pour la santé et la survie du conte (développement des publics, choix des lieux), la rencontre va se poursuivre en abordant l'évaluation plus « qualitative » des pratiques actuelles du conte et oser à plusieurs reprises mettre le doigt sur des " points sensibles » attachés à cette pratique et à son évolution récente. Échanges critiques, voire auto-critiques, du milieu ce soir-là, dans l'équilibre remarquable d'oser cette démarche et de l'oser avec grande délicatesse.

\section{L'humour}

Un premier sujet abordé a été la propension à l'humour dans le conte, et le risque encouru de tomber dans l'« humorisme ", dans la facilité. BERNARD GrondIN rappelle pourtant que l'humour fait partie, traditionnellement, du conte au Québec, et que le conte y a plutôt une fonction divertissante [NB: même si l'humour est souvent, et heureusement, présent dans les contes en général, ce n'en est pas la marque dominante dans toutes les cultures.] Grondin ajoute que, à l'instar du bouffon par exemple, l'humour, la dérision, est souvent un moyen d'exprimer le sérieux. Rapidement, la distinction est faite entre l'humour comme fin en soi (le « juste pour rire ») et l'humour comme moyen de faire circuler, efficacement, d'autres valeurs (C.-M Pons). Le « devoir faire rire » est souligné comme un risque, notamment celui de polariser (JEANMARC MASSIE) ; il est lié à la tendance actuelle du spectaculaire et du contespectacle. "Quand l'humour efface l'histoire, ça devient du stand-up comic » (J.-M Massie). 


\section{Le vedettariat}

Dans une suite logique, la discussion aborde un nouveau point : celui du statut de « vedette » qui rejoint le conteur, et les glissements que cela pose, notamment du côté des attentes du public : venir voir un conteur, plutôt que venir écouter du conte. Le conteur, comme passeur anonyme de récits, attiré par les lumières de la « star » : C.-M. Pons évoque en comparaison le passage, à la Renaissance, de l'artisan, bon ouvrier imagier à qui l'on commandait des peintures, devenant artiste en signant ses toiles; Pons souligne dans ce sens l'attrait paradoxal des conteurs factuels, qui, après avoir retrouvé l'oralité du conte en le « délivrant » de l'écrit (un des éléments déclencheurs du Renouveau du conte), ne dédaignent pourtant pas publier leurs contes aux Éditions Planète rebelle ou ailleurs, ni ne refusent une séance de signature au prochain salon du livre... Vedettarisation sans doute fort dépendante, et peutêtre incontournable, des phénomènes socio-économiques inhérents à notre modernité. JEAN-MARC MASSIE poursuit : l'important est moins de concéder à la "starification » que de savoir quoi faire de sa renommée ; s'asseoir dessus ou poursuivre, inventer, prospecter, prendre des risques (il nomme Michel Faubert en exemple).

C.-M. Pons distingue ce statut de renommée qu'on peut finalement atteindre, dans le cas de Faubert, après des années de défrichage et de travail, de celui envié par une nouvelle génération de conteurs au chemin maintenant bien ouvert : la hâte de prétendre atteindre trop vite le « temple de la renommée », d'obtenir reconnaissance aux dépens de la connaissance et du savoirfaire, de prendre le temps de mûrir. En ceci, comment voir l'avenir, la relève ?

\section{La formation}

La notion, et la question, de la formation est alors mise sur table. BERNARD GRONDIN : «Que fait-on pour les conteurs qui commencent? ». Il suggère comme solution éprouvée : celle du cercle, cercle des conteurs (plusieurs conteurs, jeunes et moins jeunes, se réunissent, content et commentent). Privilégier la formule du cercle et l'absence de «maître » (dirigeant stage ou atelier, comme on le pratique régulièrement en France). Le dispositif du cercle permet un retour critique sans qu'il s'agisse pour autant d'école. Idée du cercle ouvert ou fermé, avec ou sans public : l'applaudimètre est aussi une forme de rétroaction (LEMELIN).La formule fait écho et accord auprès des autres participants de la table : le cercle serait le modèle de formation adapté aux besoins du Québec, permettant de conserver une « liberté dans la critique » (ANDRÉ LEMELIN), une « concertation dans la critique » (JEAN-MARC MASSIE), sur fond de collégialité. Grondin précise que ce mode de formation et d'échange n'est pas destiné qu'à produire des conteurs sur scène, mais permet aussi une activité sociale, un mode d'animation auprès de certains 
groupes (évocation d'une expérience menée par la conteuse ClaUdETTE L'HEuREuX). En revanche, l'idée d'une école du conte est vite rejetée comme risque de constituer un moule (JACQUES FALQUET) ; Falquet préfère miser sur l'autoformation à travers des occasions informelles de perfectionnement, sous forme encore d'accompagnement. ANDRÉ LEMELIN rappelle qu'il n'y avait pas, traditionnellement, ni formation ni écoles du conte, mais que l'apprentissage se faisait de bouche à oreille. DENIS TRUDEL souligne l'importance d'aller voir et écouter des conteurs pour apprendre. Le consensus est assez clair autour de la table quant au choix d'une formation non régimentée ni académique au profit d'occasions d'échanges, de partage et de collégialité. Modèle en cela distinct des pratiques voisines, anglophones, suivies par les storytellers.

\section{L'appropriation du répertoire}

Face au risque d'entendre trop souvent répéter le même conte par le même conteur, distinction là encore est faite de la fréquence d'écoute : entre les proches du conte qui suivent de près les événements, mais qui sont exception, et le public en général plus occasionnel et plus aléatoire ; un même conte peut facilement être redit s'il ne s'adresse pas aux mêmes oreilles. Il appartient au conte, même, d'être répété (JACQUES FALQUET) et réécoutable " comme une chanson » (J.-M. MASSIE). Mais il y a cependant des limites à la répétition, parfois atteintes par des conteurs se produisant dans les mêmes lieux. La question de la « répétition » ouvre deux autres problèmes : celui du conte «à la mode » qui, à un moment donné, est repris par plusieurs conteurs au même moment et qui circule comme rumeur envahissante. L'autre phénomène, lié, est celui de l'emprunt par un conteur d'un conte entendu de la bouche d'un autre. Circulation en partie naturelle, il n'y a pas vraiment, traditionnellement, de copyright sur des récits souvent collectifs. En revanche, l'emprunt d'un conte de création (produit par un conteur particulier, identifiable, différent d'un conte dit traditionnel, souvent anonyme ou versé depuis longtemps dans le domaine public) ou la reprise « mot à mot » d'une version entendue posent problème. La distinction se situe autour du principe d'appropriation, c'est-à-dire la capacité par un conteur de personnaliser tel ou tel récit en circulation en produisant sa propre version, identifiable et différente des autres. C.-M. Pons fait allusion à l'une des soirées du récent festival "Les jours sont contés en Estrie " proposant le " conte de la bosse » repris successivement par quatre conteurs différents (versions hollandaise, africaine, irlandaise et ...nord-ontarienne) et qui fut un succès ; ANDRÉ LEMELIN cite le fameux « Rose Latulipe » dont on aurait repéré pas moins de deux mille versions, juste au Québec. 


\section{La tradition québécoise}

Pour Lemelin, le trait dominant de la tradition québécoise du conte est celui de la "réjouissance " (qu'il préfère à divertissement), et cette fonction d'origine du conte au Québec explique, ou justifie bien des aspects du conte contemporain, tel qu'il est majoritairement vécu au Québec. La propension à l'humour, déjà évoquée, serait héritière de ce principe de réjouissance : plutôt que d'utiliser le conte, comme l'ont fait d'anciennes cultures, à des tentatives de compréhension cosmogonique, ou fondatrice du monde, ou comme moyens édifiants ou initiatiques, le Québec, trop récent pour remonter à ces époques mythiques, use du conte comme objet de plaisir et de dérision et moyen d'occuper joyeusement la soirée et la longueur des hivers. Image du « mon oncle » animant de sa verve le temps des fêtes, ou celle du conteur embauché pour meubler les dimanches désœuvrés du camp de bûcherons, loin de toute autre source de loisir. Tradition de réjouissance aux récits facilement iconoclastes (LEMELIN) plutôt qu'enclins aux sagas respectables et transcendantes, intouchables. Fondé au temps d'une « modernité » (relativement aux longues traditions des « vieux » pays et en l'absence de traditions millénaires), le Québec, et son rapport au conte notamment, poursuit cette relation avec la légèreté et la liberté de pouvoir inventer, improviser loin des contraintes et du poids d'une histoire de références obligées. Anecdote de DenIS TRUDEL, bricolant au Japon un cerf-volant d'un morceau de soie par lui peint et à l'aide de quelques goujons de fortune sous les yeux sidérés des maîtres du Rokkaku aux modèles consacrés : image du " patenteux » refaisant le monde dans l'irrespect des règles instituées (et, en plus, ça marche !).

Le rire, l'improvisation, la récupération et le détournement délinquants de récits convenus, le refus d'une école ou d'un maître gardien du bonusage, l'irrespect et le culot d'en « lancer une » (excusez-là !) pour le seul plaisir d'en rire et de partager un bon moment, resteraient finalement les principales sources d'inspiration de nos conteurs québécois, aujourd'hui.

La première question mise sur table, celle de dresser bilan, ou portrait du conte contemporain au Québec, avait pour intention de révéler et de constater le statut particulier, dans son état et sa dynamique, de cette pratique du conte et de mieux la situer au sein du patrimoine vivant. La seconde question envisagée était celle d'évaluer, à partir de ce constat établi, la place du conte auprès du CQPV, et l'intégration du nouveau Regroupement du conte au Québec.

La question est lancée, la réponse est laissée à JEAN-MARC MASSIE, président du RcQ. Réaffirmation que le RcQ a été fondé dans le but de représenter, soutenir, promouvoir et défendre les intérêts du conte au Québec 
et qu'il a été constitué non dans l'esprit de s'opposer mais de s'allier au Conseil québécois du patrimoine vivant. Les modalités même de l'alliance entre les deux organismes est à discuter et à préciser auprès des deux conseils d'administration respectifs. Nous en saurons plus la prochaine fois. 